\section{Codebook based Direct Vector Quantization of MIMO Channel Matrix with Channel Normalization}

\author{
Bing Hui, KyungHi Chang
}

\section{ABSTRACT}

In this paper, a novel codebook generation strategy is proposed. With the given codebooks, two codeword selection procedures are proposed and analyzed for generating the quantized multiple-input multiple-output (MIMO) channel state information (CSI). Furthermore, three different quantization and normalization strategies are analyzed. The simulation results suggest that the proposed 'quantized channel generation method 2' is the best strategy to reduce the quantization and normalization errors to generate the final quantized MIMO CSI.

Key Words : Codebook, MIMO, normalization, vector quantization, quantization error

\section{Introduction}

One widely used way to generate limited feedback is by using the codebook. The LTE codebooks are designed for obtaining the benefits of multiple-input multiple-output (MIMO) technology with less complexity impact incurred through transmission and reception. None of the advanced pre-processing techniques, such as non-linear precoding and dirty-paper coding, can be implemented with the LTE codebooks which is unitary. The challenge for implementation of advanced pre-processing techniques is to obtain the
MIMO CSI at Tx side as accurate as possible under the constraint of limited feedback. In this paper, several codebook generation strategies for recovery of full channel matrix are proposed and compared to the well-known LTE codebooks. In order to reduce the quantization error, the power normalization issues are discussed and analyzed, and the best way of generating quantized MIMO channel matrix with given codebooks is suggested.

Fig. 1 shows the system model of a multi-user MIMO (MU-MIMO) employing codebook for precoding at base station (BS) side ${ }^{[1]}$, where each user has single antenna. The square MIMO channel matrix is analyzed as an example, where the number of rows equals that of columns, i.e., the number of simultaneous users equals the number of transmit antennas. In order to achieve full degree of freedom (DoF) in the perspective of MIMO capacity, we assume the MIMO channel matrix is full rank (non-singular matrix).

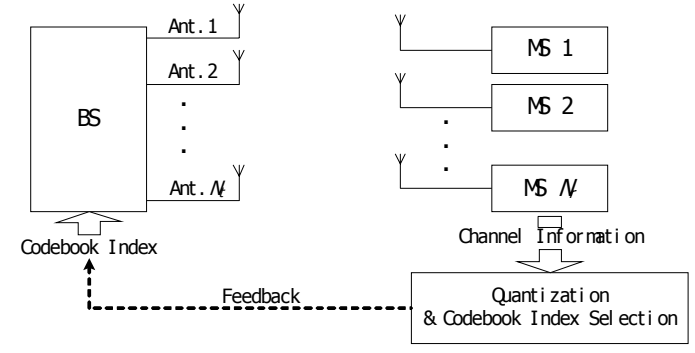

Fig. 1. Block diagram of MU-MIMO system with limited feedback.

\section{VQ of MIMO Channel Matrix}

Unlike the unitary precoding techniques ${ }^{[2]}$, our codebook generation strategy is proposed for recovery of the full MIMO channel matrix.

The generation procedure of quantized channel matrix type $\mathbf{1}$ can be described as follows.

※ This work was supported by the Industrial Strategic Technology Development Program (10041734, Development of feature-dispersive base station technology supporting multiRAT/multi-tier for next-generation integrated access system), and was also supported by (10044540, Development of small cell base station supporting IMT-Advanced TDD radio technology for evolution of TDD network) funded by the Ministry of Science, ICT and Future Planning (MSIP), Korea.

- First Author : Department of Electronic Engineering, Inha University, huibing_zxo@163.com, 정회원

- Corresponding Author : Department of Electronic Engineering, Inha University, khchang@inha.ac.kr, 종신회원 논문번호: KICS2014-01-024, Received January 29, 2014; Reviewed February 17, 2014; Accepted March 6, 2014 
1) The codewords are column vectors.

2) The quantized matrix is generated by vector quantization (VQ) of the channel matrix.

3) The quantization criteria is searching the codeword with the smallest Chordal distance from the column vectors of original channel matrix.

The above procedure is a straightforward way to generate quantized MIMO channel matrix. However, the generated quantized matrix may be a singular matrix, since multiple columns of original channel matrix may be mapped to identical codewords, which leads to the reduction the DoF.

To avoid the codeword collision problem, the quantized channel matrix type $\mathbf{2}$ is proposed.

1) Calculate the Chordal distances between the vectors of original channel matrix and codewords.

2) Choose the codeword with smallest Chordal distance as the corresponding vector in final quantized channel matrix.

3) Exclude the previous chosen codeword from the list of available codewords.

4) Repeat previous steps for the next unquantized vectors until the end.

It is obvious that this suboptimal procedure can avoid the codeword collision perfectly and the computational complexity is fixed, but the minimal quantization error can't be guaranteed.

\section{Channel Normalization}

Three quantized channel generation methods (QCGMs) are given to analyze the channel quantization and normalization errors. Define the MIMO channel matrix is $\mathbf{H}$ and the gain is,

$$
G(\mathbf{H})=\operatorname{sum}\left(\operatorname{sum}\left(\mathbf{H}^{H} \mathbf{H}\right)\right) \text {. }
$$

where $G(X)$ and $\operatorname{sum}(X)$ represent the gain and summation of elements in matrix $X$, respectively.

The strategy for QCGM 1 is given as below.

1) Codebook generation based on the un-normalized channel collections.

2) Quantization of the un-normalized CSI

$$
\mathbf{H}_{\text {quantized }}=Q\left(\mathbf{H}_{\text {unnormalized }}\right) \text {, }
$$

where $Q($.$) is quantization with codebook.$

3) Normalization of the CSI
○ Original CSI :

$$
\mathbf{H}_{C S I}=\frac{1}{\sqrt{G\left(\mathbf{H}_{\text {unnormalized }}\right)}} \cdot \mathbf{H}_{\text {unnormalized }}
$$

○ Quantized CSI :

$$
\mathbf{H}_{\text {quantized CSI }}=\frac{1}{\sqrt{G\left(\mathbf{H}_{\text {quantized }}\right)}} \cdot \mathbf{H}_{\text {quantized }}
$$

By processing the QCGM 1, the quantization error exists due to the quantization using codebook. Furthermore, since the channel gains of the original channel matrix and the quantized channel matrix are different, normalization after quantization introduces more nondeterminacy to the final generated quantized CSI $H_{\text {quantizedCSI }}$.

The strategy for QCGM 2 is given as below.

1) Codebook generation based on the normalized channel collections.

2) Quantization of the normalized CSI

$$
\mathbf{H}_{\text {quantizedCSI }}=Q\left(\mathbf{H}_{\text {normalized }}\right)
$$

Since the quantization is implemented after channel normalization, only quantization error exist by using QCGM 2.

The strategy for QCGM 3 is given as below.

1) Codebook generation based on the normalized channel collections.

2) Quantization of the normalized CSI as shown in (5)

3) Normalization of the quantized CSI

$$
\hat{\mathbf{H}}_{\text {quantizedCSI }}=\frac{1}{\sqrt{G\left(\mathbf{H}_{\text {quantizedCSI }}\right)}} \cdot \mathbf{H}_{\text {quantizedCSI }}
$$

The QCGM 3 is an improved version of QCGM 2 , and the normalization of quantized CSI $H_{\text {quantizedCSI }}$ makes that the final quantized channel matrix $\hat{H}_{\text {quantizedCSI }}$ has the channel gain of 1 . But uncertainty has been introduced by the normalization of step 3 again.

\section{Simulation Results}

Assume there is no feedback delay. The modulation is QPSK. One packet contains 100 data symbols. The fading channel is Rayleigh distributed. The linear zero-forcing (LZF) precoding with quantized MIMO CSI is performed. The codebook size is 8 ( 3 bit codebook index). Fig. 2 shows the 
errors introduced by quantization and normalization for different QCGMs. When QCGM 2 is implemented, the mean error is the smallest compared to other 2 methods. Fig. 2 indicates that the normalization of quantized channel matrix introduces more quantization error in average. Fig. 3 shows the bit error rate (BER) and packet error rate (PER) performances. Obviously, the system employing QCGM 2 shows the best performance.

Our proposed codebook generation strategy can also be used for advanced precoding techniques. Fig. 4 shows the performance comparison between the LTE codebook with unitary precoding and the proposed codebook with Tomlinson-Harashima precoding (THP $)^{[3]}$. The results are obtained in $4 \times 4$ system, and show that the implementation of LTE codebook has much worse performance. This implies that even though the significant performance gain (by the proposed codebook) is achieved at a higher cost of the number of feedback bits, the proposed codebook generation strategy shows a possible evolutionary direction for practical

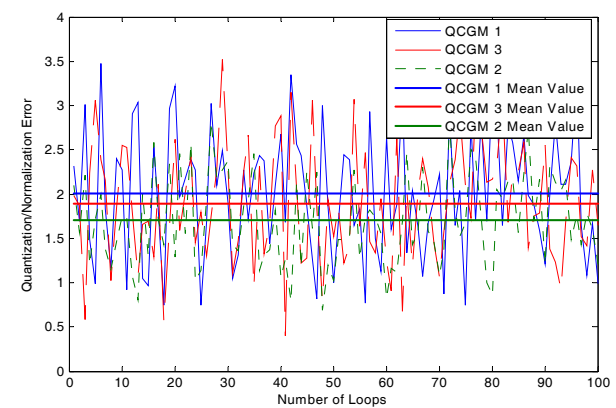

Fig. 2. Errors of different QCGMs due to quantization and normalization.

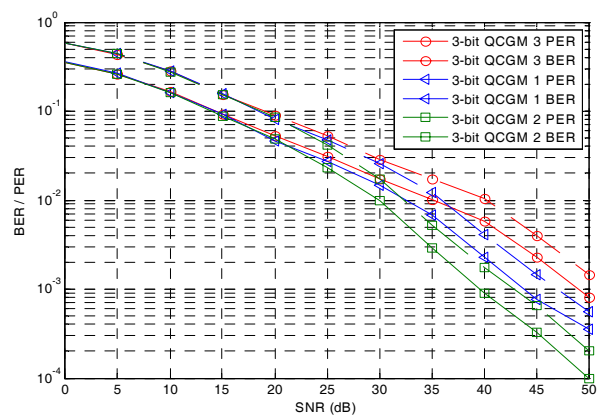

Fig. 3. BER and PER performances employing different QCGMs in $2 \times 2$ system.

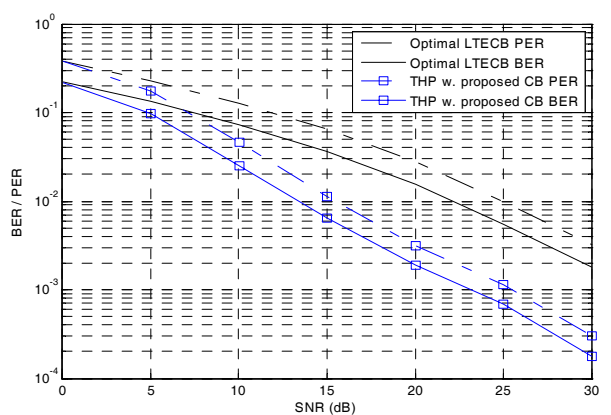

Fig. 4. Performance comparison employing LTE codebooks and general codebooks.

implementations.

\section{Conclusions}

Being different from the existing codebooks for unitary precoding, such as LTE codebooks, a general codebook generation criterion based on the minimum Chordal distance is introduced. Two codeword selection procedures and three QCGMs are proposed and compared. The simulation results show that the proposed QCGM 2 is the best strategy for quantizing MIMO CSI. Additionally, the adoption of the proposed codebook generation strategy shows a possible evolutionary direction for implementing the advanced precoding techniques for future practical applications.

\section{References}

[1] B. Hui and K. H. Chang, "On capacity of MISO beamforming with codebook based scalar quantization," in Proc. IEEE Int. Conf. Acoustics, Speech, and Signal Processing 2012 (IEEE ICASSP 2012), pp. 3505-3508, Kyoto, Japan, Mar. 2012.

[2] D. J. Love and R. W. Heath, "Limited feedback unitary precoding for spatial multiplexing systems," IEEE Trans. on Inf. Theory, vol. 51, no. 8, pp. 2967-2976, Aug. 2005.

[3] M. Mohaisen, D. K. Han, B. Hui, K. H. Chang, and B. T. Koo, "Vector perturbation techniques with block diagonalization for multi-user MIMO systems," J. KICS, vol. 35, no. 10, pp. 922-929, Oct. 2010. 